\title{
Extracting, storing, and transporting whole blood DNA under tropical conditions
}

\author{
I Bates, G Bedu-Addo, T R Rutherford
}

\begin{abstract}
A simple and robust technique for the extraction of DNA under tropical field conditions is described. It requires minimal equipment and is based on lysing cells in whole blood and precipitating the nuclei containing the DNA by centrifugation. The DNA solution can be stored in guanidinium buffer for many months without being refrigerated. Further purification of the DNA can then be carried out in a laboratory with facilities for ultracentrifugation by banding the DNA through cesium chloride. This method yields DNA of sufficient quality and purity for Southern blotting and probing and alleviates the need to transport whole blood between different countries and laboratories.
\end{abstract}

The application of DNA analysis to the study of tropical diseases is difficult because of problems with blood transportation and storage, or DNA extraction under field conditions. We have developed a simple, reliable method of extracting DNA from whole blood which can be carried out in a small hospital laboratory. DNA is obtained in a form suitable for storage without refrigeration at ambient tropical temperatures for many months.

\section{Methods and results}

At least $5 \mathrm{ml}$ anticoagulated blood are mixed with $50 \mathrm{ml}$ cell lysis buffer (figure). Nuclei are isolated by centrifugation at $2500 \mathrm{rpm}$ for 10 minutes and the supernatant discarded. The nuclear pellet is vigorously resuspended in the last drop of liquid and mixed with 1-2 ml nuclear lysis buffer (figure). The DNA solution is transferred to a $2 \mathrm{ml}$ tube and can be stored in this form for many months at ambient temperatures before purification in the United Kingdom.

Further purification is achieved by layering the nuclear lysate over a $3 \mathrm{ml}$ pad of $1 \mathrm{~g} / \mathrm{ml}$ cesium chloride in $0.1 \mathrm{M}$ EDTA and centrifuging at $32000 \mathrm{rpm}$ for 16 hours. ${ }^{1}$ The DNA band is removed with a wide bore pipette and extensively dialysed against $10 \mathrm{mM}$ TRIS $1 \mathrm{mM}$ EDTA (pH 7.5) $(1 \times \mathrm{TE})$ to remove traces of guanidinium and cesium chloride.

The level of degradation of the DNA was assessed by electrophoresing $0.5 \mu \mathrm{g}$ DNA through an agarose gel and observing the size of the DNA after staining with ethidium bromide. In most samples only DNA of large molecular weight is present. A few samples contain small amounts of low molecular weight DNA, shown by smearing on the gel, and are therefore slightly degraded. Nevertheless, even in samples which exhibit some degradation the DNA is sufficiently pure to permit endonuclease digestion with enzymes, such as Hind III, Bam H1, providing 1-4 mM spermidine are added to the manufacturer's recommended buffers. ${ }^{2}$ The samples can be Southern blotted and reproducible restriction fragments are seen for each sample when probed for the heavy chain region of the immunoglobulin gene.

\section{Constituents of buffers}

Cell lysis buffer $3 \mathrm{ml} 1 \mathrm{M}$ TRIS ( $\mathrm{pH} 7 \cdot 5$ ) $32.9 \mathrm{~g}$ sucrose $1.5 \mathrm{ml} 1 \mathrm{M}$ magnesium chloride $3 \mathrm{ml}$ Triton $\mathrm{X}-100$ Water to $300 \mathrm{ml}$

This can be made up at 10 times stock strength, omitting the sucrose, and reconstituted in the field laboratory with water and domestic sugar to reduce the volume of reagents transported between laboratories.

Nuclear lysis buffer $50 \mathrm{~g}$ guanidinium thiocyanate $0.5 \mathrm{~g}$ sodium- $\mathrm{N}-$ lauryl sarcosinate $2.5 \mathrm{ml} 1 \mathrm{M}$ sodium citrate ( $\mathrm{pH} 7 \cdot 0)$ $0.7 \mathrm{ml}$ 2-mercaptoethanol Water to $100 \mathrm{ml}$

We have used this method to extract and purify DNA from $2220 \mathrm{ml}$ blood samples from Ghanaian patients with a variety of haematological disorders including chronic leukaemias. An average of $680 \mu \mathrm{g}$ DNA was obtained from each sample (range 13-324 $\mu \mathrm{g}$ ), the largest yields coming from leukaemic patients. Eighty per cent of samples yielded over $30 \mu \mathrm{g}$ DNA, and even in samples containing less DNA, there was adequate material for 1-3 Southern blots. Specimens took 1.58.5 months (average 3.8 ) to reach our United Kingdom laboratory during which time they 
were not refrigerated but stored and transported at the ambient Ghanaian temperature.

An advantage of this method is that it alleviates the need to transport whole blood between laboratories. This method almost certainly renders the samples non-infective for lipid-enveloped viruses such as HIV and hepatitis $B$, because guanidinium thiocyanate is a powerful protein denaturant used for extracting RNA from tissues rich in ribonucleases. ${ }^{3}$ Triton $\mathrm{X}-100$ is also a potent nonionic surfactant which, in combination with a solvent, has been shown to produce more than $10^{11}$-fold reduction in $\mathrm{HIV}-1$ infectivity. ${ }^{4}$
These substances disrupt the viral membranes, essentially dissociating the viral genome from its receptor, making it incapable of infecting host cells.

T:ıis work was supported by the Wellcome Trust.

1 Young $\mathrm{H}$. Recovery of DNA from guanidinium isothiocyanate-cesium chloride gradients. Focus 1984;6:11.

2 Bouche J. The effect of spermidine on endonuclease inhibition by agarose contaminants. Analyt Biochem 1981; 115:42-5.

3 Chirgwin J, Przybyla AE, MacDonald RJ, et al. Isolation of biologically active ribonucleic acid from sources enriched biologically active ribonucleic acid from sources
in ribonuclease. Biochemistry 1979;18:5294-99.

in ribonuclease. Biochemistry 1979;18:5294-99.
4 Piszkiewicz D, Chong-Son S, Tondrau SC. Inactivation and removal of HIV in monoclonal purified anti-hemophilic factor (human). Thromb Res 1989;55:627-34.

\title{
Use of leucocyte alkaline phosphatase (LAP) score in differentiating malignant from benign paraproteinaemias
}

\author{
G Majumdar, M Hunt, A K Singh
}

\begin{abstract}
The leucocyte alkaline phosphatase (LAP) score of peripheral blood neutrophils was examined in 20 patients with multiple myeloma and compared with the score in 18 patients with monoclonal gammopathy of undetermined significance (MGUS). The mean ( $95 \%$ confidence limit) LAP score in those with multiple myeloma was 186 (169-218) compared with $92(64-120)$ in the MGUS group. In the multiple myeloma group all but one patient had a high LAP score, irrespective of disease. No cause for raised LAP, such as infection, was present in any of the patients with multiple myeloma. In the MGUS group six patients had a raised LAP score; in two of them another cause for such a rise was present (autoimmune haemolytic anaemia and primary thrombocythaemia). In neither group did the LAP score correlate with duration of the disease, bone marrow plasma cell count, paraprotein concentration, haemoglobin, total white cell or neutrophil count.

It is concluded that a normal LAP count in patients with paraproteinaemia suggests a benign condition, but a raised count does not indicate a malignant condition.
\end{abstract}

Monoclonal gammopathy is a common disorder, especially in the elderly, but only a small percentage of these patients have overt multiple myeloma or related conditions at the time of diagnosis. The rest are diagnosed as having MGUS and usually followed up for an indefinite period as a significant proportion of them progress to multiple myeloma and related malignant disorders. ${ }^{1}$ Several tests have been proposed for differentiating malignant from benign paraproteinaemia but none has been found to be fully reliable. The LAP score has been recommended as a useful test for such differentiation. ${ }^{2}$ We examined the LAP score in patients with multiple myeloma and MGUS to assess the value of this test in differentiating malignant from benign paraproteinaemias.

\section{Methods}

Twenty patients $(11$ men, nine women, mean age 67 years) with multiple myeloma diagnosed by the standard criteria ${ }^{3}$ were included in this study. Four were newly diagnosed, 11 were in the plateau phase and five were in relapse. The MGUS group comprised 18 patients (10 men, eight women, mean age 63 years) with paraproteinaemia who did not fulfil the diagnostic criteria for multiple myeloma and were followed up for at least 24 months without any change in disease course. None in either group had a raised white cell count at the time of the present investigation. Blood films were made at the time of routine follow up and were stained for LAP by using a commercial kit (Diagnostica Merck). Scoring

Correspondence to: Dr Majumdar

Accepted for publication 27 December 1990 